\title{
Emotional Intelligence is a Protective Factor for Suicidal Behavior
}

\section{Citation}

Cha, Christine B. and Marin K. Nowak. 2009. Emotional intelligence is a protective factor for suicidal behavior. Journal of the American Academy of Child and Adolescent Psychiatry 48: 422-430.

\section{Published Version}

doi:10.1097/CHI.0b013e3181984f44

\section{Permanent link}

http://nrs.harvard.edu/urn-3:HUL.InstRepos:4133810

\section{Terms of Use}

This article was downloaded from Harvard University's DASH repository, and is made available under the terms and conditions applicable to Open Access Policy Articles, as set forth at http:// nrs.harvard.edu/urn-3:HUL.InstRepos:dash.current.terms-of-use\#OAP

\section{Share Your Story}

The Harvard community has made this article openly available.

Please share how this access benefits you. Submit a story.

\section{Accessibility}


Words: 6054

Tables: 2

Figures: 1

\title{
Emotional Intelligence is a Protective Factor for Suicidal Behavior
}

\author{
Christine B. Cha, B.A. \\ Matthew K. Nock, Ph.D.
}

From the Department of Psychology, Harvard University, Cambridge, MA.

Correspondence to Matthew K. Nock, Ph.D., Department of Psychology, Harvard University, 33

Kirkland Street, Cambridge, MA 02138, e-mail: nock@wjh.harvard.edu.

This research was supported by grants from the William F. Milton Fund, and the William A.

Talley Fund of Harvard University. 


\begin{abstract}
Objective: Little is known about what factors protect against the occurrence of suicide ideation and attempts. We tested whether emotional intelligence (EI) - the ability to perceive, integrate, understand, and manage one's emotions - decreases the likelihood of suicide ideation and attempts among those at risk.
\end{abstract}

Method: Adolescents $(N=54)$ aged 12-19 were recruited from local psychiatric clinics and the community to participate in this cross-sectional laboratory-based study. Analyses examined whether the relations between childhood sexual abuse (CSA) and suicide ideation and attempts were moderated by adolescents' EI. These constructs were assessed using self-report, structured interviews, and performance-based tests, respectively.

Results: Analyses revealed that EI is a protective factor for both suicide ideation and attempts. Specifically, CSA was strongly predictive of these outcomes among those with low EI, weakly predictive among those with medium EI, and completely unrelated among those with high EI. Follow-up analyses revealed that the protective effect of EI was driven primarily by differences in Strategic EI (i.e., ability to understand and manage emotions) but not Experiential EI (i.e., ability to perceive emotions and integrate emotions into thoughts).

Conclusions: This study provides preliminary evidence that EI is a protective factor for suicide ideation and attempts. Important next steps include testing the moderating influence of EI on a wider range of stressful life events and self-injurious behaviors, as well as conducting experimental studies to determine whether enhancing EI decreases the subsequent occurrence of these behavior problems.

Key Words: suicide, suicidal ideation, emotional intelligence, sexual abuse 
Suicidal behaviors, which include suicide ideation and attempts, are prevalent and dangerous behavior problems around the world, particularly among adolescents. ${ }^{1-3}$ Although service utilization among those experiencing suicidal behaviors has increased significantly over the past decade, the rates of non-fatal suicidal behaviors have not decreased. ${ }^{4}$ These findings underscore the importance of identifying factors that decrease the risk of suicidal behaviors and provide the motivation for the current study.

Research on suicidal behaviors has primarily aimed at identifying risk factors, such as the experience of childhood maltreatment and other adverse life events (e.g., interpersonal loss, school or work problems). ${ }^{5-7}$ However, most people who experience stressful life events never engage in suicidal behaviors. This raises the important question of what factors might protect against the occurrence of these outcomes. Protective factors are those that decrease the probability of a negative outcome among those at risk. ${ }^{8}$ It is important to note that a protective factor is not merely defined by the absence of a risk factor. Rather, it is a third variable that modifies the strength or direction of the relation between a risk factor and outcome. For instance, several recent studies suggest that genetic and neurobiological factors can buffer the influence of stressful life events on the likelihood of suicide attempts. ${ }^{9-11}$ Other research suggests that environmental factors such as reduced accessibility to firearms, ${ }^{12,13}$ religious affiliation, ${ }^{14,15}$ and social support ${ }^{16-18}$ may moderate the influence of stressful life events on suicide risk. The study of psychological protective factors for suicidal behaviors represents an important research direction given that such factors are likely to be more easily modified than other moderators identified by prior work (e.g., genetic polymorphisms, presence of social support). 
Psychological theories of suicide suggest that people engage in suicidal behaviors due to an inability to tolerate or modulate the experience of negative affect. ${ }^{19,20}$ It is therefore possible that those who are especially adept at perceiving, integrating, understanding, and managing their emotions would be at reduced risk for suicidal behaviors in response to stressful life events. Accordingly, the current study was designed to test whether stronger abilities in each of these domains are protective against suicidal behaviors in the presence of significant stressful life events.

In the current study, stressful life events were operationalized as the experience of childhood sexual abuse (CSA). This decision was based on several factors. First, prior studies testing potential moderators of the relation between life stressors and suicide attempts and related forms of psychopathology (e.g., depression) have used childhood maltreatment ${ }^{21}$ and CSA in particular $^{11}$ as a measure of stressful life events. The assessment of CSA in the current study therefore facilitates comparisons across studies. Second, the association between CSA and suicidal behaviors has been replicated across many studies. ${ }^{22,23}$ CSA is a stronger risk factor for suicidal behaviors than other forms of childhood maltreatment ${ }^{24}$ or childhood adversities (e.g., loss of caregiver, family violence, parental psychopathology). ${ }^{25,26}$ Third, past work has reported that history of CSA affects the transmission of suicide risk from parents to offspring, ${ }^{27,28}$ further highlighting need to better understand this risk factor.

The way that emotions are experienced and their influence on thoughts and behaviors has become the focus of increasing interest and study in psychology and psychiatry. ${ }^{29,30}$ Most research in this area has focused on the construct of emotion regulation; although, this construct has been defined and measured in very different ways across studies. ${ }^{31}$ In the current study, we sought to examine the moderating effect of a broad range of emotional processes or abilities, 
including (but not limited to) the way in which people manage their emotional experiences. To do so, we examined the moderating effect of Emotional Intelligence (EI) - a multi-dimensional construct that refers to the ability to reason about emotions, as well as the ability to use emotions in guiding one's reasoning and behavior. ${ }^{32-35}$ EI, which has been the focus of a substantial body of research over the past two decades, can be decomposed into two main abilities (each composed of two sub-branches). Strategic EI is defined as the ability to understand and manage emotions (e.g., understanding what feelings are expected to emerge in response to different events, and knowing how to regulate emotions to help attain one's goals). In contrast, Experiential EI is defined as the ability to perceive emotions and use them to facilitate thought (e.g., accurately reading facial expressions and integrating emotions into thoughts). Strategic EI is considered to be a more developmentally complex ability that is involved in managing one's emotions in order to facilitate problem-solving and decision-making, whereas Experiential EI is considered to represent lower level abilities. ${ }^{32-35}$

Some prior research has linked problems with EI and the experience of clinical behavior problems. For instance, several aspects of self-reported EI similar to Experiential (e.g., perceiving emotions) and Strategic (e.g., managing others' emotions) have been reported to moderate the relation between stress and the outcomes of hopelessness, depression, and suicidal ideation. ${ }^{36}$ The current study extends this earlier work by using a more comprehensive and rigorous measure of EI and by examining the relation between EI and suicidal behaviors. The goal of the current study was to test the primary hypothesis that EI moderates the relation between CSA and the experience of suicidal behaviors such that higher EI will be associated with lower levels of suicide ideation and suicide attempts. Given that prior studies suggest that suicidal behavior may result from problems regulating one's negative emotions, ${ }^{19,20}$ a secondary 
hypothesis was that Strategic EI would be a particularly important moderator of the relation between CSA and suicidal behavior.

\section{Participants}

\section{Method}

Fifty-four (46 female) adolescents (age in years: $M=17.30, S D=1.92$, range 12-19), 31 with a recent (i.e., past year) history of suicide ideation (57.4\%) and/or attempts (14.8\%) and 23 with no recent history, participated in the current study. Suicidal and non-suicidal groups were matched on age, sex, and race/ethnicity to rule out the possibility that any observed effects are due to these factors. They were recruited using study advertisements placed in local psychiatric clinics, newspapers, community bulletin boards, and the internet. We focused on adolescence because of the increased risk of self-injurious behavior during this developmental period. ${ }^{2,3}$ These 54 participants were drawn from a larger sample of 94 adolescents who participated in a laboratory-based study of nonsuicidal self-injury. ${ }^{37-39}$ This study is reported separately due to the novel hypothesis and constructs (i.e., EI, suicide ideation and attempts) that were the focus of current analyses, and because the EI measure was added to the study after it had begun and so was only administered to these 54 adolescents. Adolescents in the current sample did not differ from the 40 excluded on any of the study variables measured. The current sample size provides adequate statistical power to detect large effects (power=.95) and some medium effects (power=.62) using two-tailed tests with alpha set at .05. Ethnicity of the participants was selfidentified as European American (79.6\%), Hispanic (5.6\%), Asian American (3.7\%), African American (1.9\%), and Other (9.2\%).

Measures

Childhood Trauma Questionnaire [CTQ]. The $\mathrm{CTQ}^{40}$ is a 28 -item $(<5 \mathrm{~min})$ self-report measure of childhood and adolescent maltreatment, including: physical abuse, sexual abuse, 
emotional abuse, physical neglect, and emotional neglect. For the purposes of this paper, we focus on CSA alone - calculated by adding up responses to its five corresponding items. Items are rated on a five-point scale $(0=$ never true, $5=$ very often true $)$. The CTQ has demonstrated high internal consistency, convergent and discriminant validity, as well as good sensitivity and specificity for all forms of maltreatment among a clinical sample of adolescents. ${ }^{40}$ The CSA subscale demonstrated excellent internal consistency $(\alpha=.89)$ in the current sample. Overall, $20.4 \%$ of the current sample endorsed at least one item from the CSA subscale.

Mayer-Salovey-Caruso Emotional Intelligence Test: Youth Version, Research Version 1.0 [MSCEIT:YV-R]. The MSCEIT:YV-R ${ }^{41}$ is a 180-item (15-20 minute), performance-based measure that assesses the two areas (Strategic vs. Experiential) and four branches of EI, including: Understanding and Managing Emotions, which together define Strategic EI, as well as Perceiving Emotions and Facilitating Thought, which together define Experiential EI. The Understanding Emotions section requires the participant to read a description of a blend of emotions (e.g., "When you have something really nice, and then you lose it, you end up feeling...”) and to select the answer choice representing most accurate complex feeling (e.g., jealous, disgusted). The Managing Emotions section requires the participant to read brief scenarios (e.g., "A boy received some very sad news. He wants to feel happy before going to a fun party. How helpful would each of the following be in getting the boy to feel happy?") and to rank along a standardized scale the degree of constructiveness of each presented possible solution. The Perceiving Emotions section requires the participant to rank along a standardized scale the extent to which photographed facial expressions suggest certain emotions (e.g., surprise, disgust). Finally, the Facilitating Thought section requires the participant to rank along a standardized scale the extent to which a particular emotion (e.g., "feeling sad") is similar to 
various tactile, color, and taste sensations (e.g., warm, heavy, dark, cold). Completed MSCEIT:YV-R packets were sent to Multi-Health Systems Inc. to be scored using the expert scoring criteria, where points were awarded according to a scoring key agreed upon by the authors of the MSCEIT:YV-R. Specifically, the MSCEIT:YV-R items were scored according to the pre-set scale ranging from 0 (less correct) to 2 (more correct), and the sum of the respective items yielded branch, area, and total MSCEIT:YV-R scores. Although other psychometric properties of the MSCEIT:YV-R have not yet been published, the original MSCEIT has demonstrated excellent split-half and test-retest reliability, content and structural validity, as well as discriminant validity in relation to other cognitive abilities and personality traits. ${ }^{42-44}$ Self-Injurious Thoughts and Behaviors Interview [SITBI]. The $\mathrm{SITBI}^{45}$ is a structured interview (3-15 minutes) that assesses presence, frequency, and other characteristics of a broad range of self-injurious thoughts and behaviors, including suicide ideation ("Did you ever have thoughts of killing yourself?") and attempt (“Did you ever make an actual attempt to kill yourself?"). In the current study, we focused on items measuring the frequency of suicide ideation and attempts in the past year. The SITBI has demonstrated reported strong inter-rater reliability, test-retest reliability, and concurrent validity. ${ }^{45}$ The SITBI was administered by trained master's- and doctoral-level researchers, as well as by closely supervised bachelor's-level research assistants. At the conclusion of each interview, each participant underwent a risk assessment and received a referral for clinical services when indicated.

\section{Procedures}

Participants who responded to the study advertisements were invited to the laboratory and provided with a complete description of the study. Written informed consent was obtained, with parental consent obtained for participants $<18$ years. Assessments were completed during a 
baseline laboratory assessment, for which participants received $\$ 100$. Because the MSCEIT:YV-R was incorporated into the larger study shortly after it had begun, MSCEIT:YV-R data were obtained from the first 11 participants (paid an extra $\$ 10$ ) by sending them this measure via postal mail with a return-postage envelope. MSCEIT:YV-R scores did not significantly differ between these 11 participants and the 43 who completed this measure in the laboratory. All procedures were approved by the university's institutional review board.

\section{Data Analysis}

Before conducting analyses, suicide ideation and attempt variables were adjusted to reduce the influence of outliers. Following the recommendations of Tabachnick and Fidell, ${ }^{46}$ we re-assigned outliers less extreme values (i.e., within 2 standard deviations of the mean) that retained their relative standing in the distribution (i.e., still the highest scores on each variable) and variables that were not normally distributed were transformed to more closely approximate normality. ${ }^{46}$ Next, we tested the magnitude of the relations between CSA, EI, and suicide ideation and attempts using correlations. We then tested whether the relations between CSA and suicidal behaviors were moderated by EI following the recommendations for testing moderation and conducting post-hoc probing. ${ }^{47,48}$ Specifically, the CSA and EI variables were centered prior to computing the interaction variable. The moderation models were then examined through hierarchical linear regression analyses, entering the centered CSA and EI variables in the first step and the interaction variable in the second step. Significant interactions were plotted and probed through simple slope analyses ${ }^{47,48}$ using conditional variables computed based on high (+1SD), medium, and low (-1SD) moderator (i.e., EI) values. 


\section{Results}

Relations between EI, CSA, and Suicidal Behaviors

Descriptive statistics and correlations between EI, CSA, and suicidal behaviors are presented in Table 1. As expected, CSA was significantly correlated with both suicide ideation and attempts. EI was not significantly associated with CSA or with suicidal behaviors, with the correlations among these constructs representing mostly small effects. This means that EI is not merely a correlate of, or risk factor for, CSA and suicidal behaviors. Although the absence of significant associations between EI and these other variables was not hypothesized, this pattern

of findings strengthens the potential argument for moderation. ${ }^{49}$ Is EI a Protective Factor for Suicidal Behaviors?

Consistent with our primary hypothesis, overall EI significantly moderated the relation between CSA and both suicide ideation and attempts (Table 2). As presented in Figure 1a, CSA was strongly associated with suicide ideation for participants with low EI, whereas this relation was weaker for those with medium scores on EI, and completely absent for those with high EI. The same pattern was revealed for suicide attempts.

In order to better understand which aspects of EI were protective for suicidal behavior, we separately tested the moderating effects of Strategic and Experiential EI. In support of our secondary hypothesis, analyses revealed that Strategic EI significantly moderated the relation between CSA and both suicide ideation and attempts (Table 2). As presented in Figure 1b, CSA was strongly associated with suicide ideation for participants with low Strategic EI, whereas this relation was weaker for those with medium scores on Strategic EI, and completely absent for those with high Strategic EI. The same pattern was again revealed for suicide attempts. 
Experiential EI did not significantly moderate the relations between CSA and suicide ideation or attempts (Table 2 and Figure 1c).

\section{Discussion}

The goal of this study was to determine whether EI is a protective factor for suicidal behaviors among adolescents who have experienced significant life stressors. Consistent with our hypotheses, results revealed that EI moderates the relation between retrospectively reported CSA and past year suicide ideation and attempt, such that there is a strong relation between CSA and these suicidal behaviors among adolescents with low EI; a weaker relation among those with medium EI; and no significant relation among those with high EI. Further analyses revealed that Strategic EI drove the overall protective effect, and Experiential EI did not emerge as a significant protective factor for either suicide ideation or attempts. Several aspects of these findings warrant additional comment.

The specificity of the observed protective effect of Strategic EI (but not Experiential EI) for suicidal behaviors is an important strength of this study. The fact that effects were replicated across both suicide ideation and attempts increases confidence in the reliability of these findings. These results are consistent with those from randomized controlled trials of cognitive therapy and dialectical behavior therapy (both of which include components aimed at enhanced emotion understanding and management) for the treatment of self-injurious behaviors, which cause

decreases in suicide attempts. ${ }^{50,51}$ Taken together, these findings point toward the importance of abilities for understanding and managing emotions in the treatment and prevention of suicidal behaviors. Although studies on the treatment of suicide have not typically made explicit mention of EI, the constructs targeted in these treatments (e.g., emotion regulation skills, problem-solving skills) are similar to those studied by EI researchers. On balance, it is notable that we did not 
find a statistically significant association between Strategic EI—whose managing emotions branch has been equated to emotion regulation ${ }^{52}$-and either CSA or suicidal behaviors, despite prior findings indicating that emotion regulation is correlated with these variables. ${ }^{19,20}$ Two aspects of this finding are notable. First, we did observe consistent small-to-medium associations among these variables $(r \mathrm{~s}=-.15$ to -.19$)$ and so lack of statistical significance is partly the result of our lack of power for detecting such effects. Second, differences in associations with emotion regulation-related constructs may be due in large part to variations in the way such constructs are defined and measured. In the current study, we used the construct of Strategic EI and measurement strategy provided by the MSCEIT, which assesses knowledge about effective emotion regulation strategies, but not their actual use. Prior studies have found that although self-injurers and non-injurers are equally capable of proposing effective solutions to potential interpersonal problems, self-injurers are significantly worse at the selection and performance of solutions. ${ }^{38}$ It is important that future work in this area attend carefully to differences in the terms, definitions, and measurement strategies used.

Nevertheless, some research suggests that EI is indeed a malleable target of change. One example of this has been demonstrated by Eack and colleagues, ${ }^{53}$ reporting that Cognitive Enhancement Therapy ${ }^{54}$ improves Strategic EI among patients with schizophrenia. Despite these findings, the majority of therapeutic approaches for suicide attempters emphasize improving Experiential EI (e.g., clarification of emotions experienced). ${ }^{55}$ Studies are sorely needed to test the potential benefit of targeting Strategic EI in intervention and prevention programs. ${ }^{35,56}$ Identifying EI as a protective factor represents a unique and important contribution to research on suicidal behaviors. The identification of risk factors has allowed research, treatment, and prevention efforts to isolate at-risk (e.g., sexually abused) populations; little has been done 
beyond this, as such risk factors often are not malleable. Reports on protective factors would move efforts forward by specifying what factors might keep adolescents from engaging in suicidal behaviors, thus identifying potential targets for intervention and prevention efforts. The existing literature on potential protective factors primarily pertains to religious beliefs and practice, ${ }^{14,15}$ accessibility to weapons, ${ }^{12,13}$ and social support, ${ }^{16-18}$ and very few studies explore psychological protective factors. One psychological factor explored by Linehan and colleagues ${ }^{57}$ was placing importance on reasons for living (i.e., adaptive beliefs and expectations), and few recent efforts have been made to explore resilience to suicide. ${ }^{58,59}$ The current study extends knowledge about what psychological factors protect against risk for suicidal behaviors and provide a new direction in this developing research area.

These findings also add to the growing literature on the importance of EI, and especially to earlier findings on the particular importance of understanding and managing emotions. Earlier evidence suggests that the ability to manage emotions is associated with prosocial tendencies, positive peer nominations ${ }^{52,60}$ and higher quality of social interactions. ${ }^{61}$ Strategic EI abilities have also been shown to relate to aspects of romantic partners' support style. ${ }^{62}$ These earlier findings suggest that Strategic EI may influence suicidal behavior through several different social and emotional mechanisms. Overall, the converging evidence regarding Strategic EI provides an exciting point of departure for future research on the factors that may protect against suicidal behaviors.

There are several limitations to this study. First, our sample was relatively small and so the results are based on a limited sample of episodes of suicide ideation, attempts and CSA. Our sample also consisted mostly of European American adolescent girls who were willing to participate in a research study, which may limit the generalizability of our findings. Moreover, 
because participants were recruited for a larger study of nonsuicidal self-injury, many of those with suicide ideation and all of those with suicide attempts in the current sample also had a history of nonsuicidal self-injury. These issues may limit the generalizability of the findings and it will be important to replicate these findings in a larger and more diverse sample. Second, the current study did not control for other psychological factors (e.g., personality traits, IQ) that might contribute to the effect of EI. Controlling for such factors may have strengthened our findings; however, it is worth noting that personality and IQ have been shown to relate specifically to self-reported EI and not with performance-based EI, ${ }^{63}$ and so it is unlikely that these factors would have changed the observed effects. Third, the reports of childhood CSA are based on retrospective self-report. Recent frequency of suicide ideation and attempts was also retrospectively reported. As a result, it is possible that participants inaccurately recalled or reported CSA or suicidal behaviors. Prior work suggests that although there is error in long-term retrospective reporting of past events, risk estimates for suicide attempts (e.g., odds ratios) based on self-report data compared to official records are fairly similar. ${ }^{64}$ Moreover, such risk estimates for suicide attempts based on retrospective recall have been found to be fairly stable over time. ${ }^{65}$ Nevertheless, future studies should measure CSA and suicidal behaviors prospectively and using methods that do not rely exclusively on self-report. ${ }^{37}$

Given these limitations, the results of this study should be considered preliminary evidence for the protective effect of EI that enhance our understanding of suicidal behaviors but also raise key issues to address in future research. First, it is important to replicate these findings among a larger, more diverse sample. Doing so would support the generalizability of these findings. Second, studies are needed to test prospectively whether EI protects against the effects of co-occurring stressors (e.g., poor-functioning family). ${ }^{66}$ In a related vein, future research 
should assess whether EI buffers the effect of any mechanisms of CSA traumatization ${ }^{67,68}$ or any subsequent risk factors for suicide (e.g., psychopathology). ${ }^{26,69}$ Testing the degree of generalizability to other stressors and clinical risk factors is a necessary step to clarifying the nature of relations identified here. Third, it would be fruitful to test how EI works with other potential protective factors (e.g., social support) to reduce suicide risk. Studies also are needed to test the relations between EI and potential genetic ${ }^{9,11}$ and neurobiological predispositions to suicidal behaviors. ${ }^{10}$ The identification of additional protective factors and the subsequent development of more sophisticated theoretical and empirical models represent much needed directions in the effort to prevent these prevalent and harmful behavior problems. 


\section{References}

1. Hawton K, Rodham K, Evans E. By Their Own Young Hand: Deliberate Self-Harm and Suicidal Ideas in Adolescents. London: Jessica Kingsley Publishers; 2006.

2. Nock MK, Borges G, Bromet EJ, et al. Cross-national prevalence and risk factors for suicidal ideation, plans, and attempts. Br J Psychiatry. 2008;192:98-105.

3. Nock MK, Borges G, Bromet EJ, Cha CB, Kessler RC, Lee S. The epidemiology of suicide and suicidal behavior. Epidemiol Rev. 2008;doi: 10.1093/epirev/mxn002

4. Kessler RC, Berglund P, Borges G, Nock MK, Wang PS. Trends in suicide ideation, plans, gestures, and attempts in the United States, 1990-1992 to 2001-2003. JAMA. $2005 ; 293: 2487-2495$.

5. Brent DA, Baugher M, Bridge J, Chen T, Chiappetta L. Age- and sex-related risk factors for adolescent suicide. J Am Acad Child Adolesc Psychiatry. 1999;38(12):1497-1505.

6. Dube SR, Anda RF, Felitti VJ, Chapman DP, Williamson DF, Giles WH. Childhood abuse, household dysfunction, and the risk of attempted suicide throughout the life span: findings from the Adverse Childhood Experiences Study. JAMA. 2001;286(24):30893096.

7. Gould MS, Greenberg T, Velting DM, Shaffer D. Youth suicide risk and preventive interventions: a review of the past 10 years. J Am Acad Child Adolesc Psychiatry. $2003 ; 42(4): 386-405$.

8. Kazdin AE. Research Design in Clinical Psychology. 4th ed. Boston, MA: Allyn \& Bacon; 2003.

9. Caspi A, Sugden K, Moffitt TE, et al. Influence of life stress on depression: moderation by a polymorphism in the 5-Htt gene. Science. 2003;301(5631):386-389. 
10. Mann JJ. Neurobiology of suicidal behaviour. Nat Rev Neurosci. 2003;4:819-828.

11. Perroud N, Courtet $\mathrm{P}$, Vincze I, et al. Interaction between BDNF Val66Met and childhood trauma on adult's violent suicide attempt. Genes Brain Behav. 2008;7(3):314322.

12. Brent DA, Perper J, Moritz G, Baugher M, Allman C. Suicide in adolescent with no apparent psychopathology. J Am Acad Child Adolesc Psychiatry. 1993;32:494-500.

13. Shenassa ED, Rogers ML, Spalding KL, Roberts MB. Safer storage of firearms at home and risk of suicide: A study of protective factors in a nationally representative sample. $J$ Epidemiol Community Health. 2004;58:841-848.

14. Dervic K, Oquendo MA, Grunebaum MF, Ellis S, Burke AK, Mann JJ. Religious affiliation and suicide attempt. Am J Psychiatry. 2004;161(12):2303-2308.

15. Greening L, Stoppelbein L. Religiosity, attributional style, and social support as psychosocial buffers for African American and white adolescents' perceived risk for suicide. Suicide Life Threat Behav. 2002;32(4):404-417.

16. Borowsky IW, Ireland M, Resnick MD. Adolescent suicide attempts: risks and protectors. Pediatrics. 2001;107(3):485-493.

17. O'Donnell L, O'Donnell C, Wardlaw DM, Stueve A. Risk and resiliency factors influencing suicidality among urban African American and Latino youth. Am J Community Psychol. 2004;33(1-2):37-49.

18. Resnick MD, Bearman PS, Blum RW, et al. Protecting adolescents from harm. Findings from the National Longitudinal Study on Adolescent Health. JAMA. 1997;278(10):823832. 
19. Lynch TR, Cheavens JS, Morse JQ, Rosenthal MZ. A model predicting suicidal ideation and hopelessness in depressed older adults: the impact of emotion inhibition and affect intensity. Aging \& Mental Health. 2004;8(6):486-497.

20. Zlotnick C, Donaldson D, Spirito A, Pearlstein T. Affect regulation and suicide attempts in adolescent inpatients. J Am Acad Child Adolesc Psychiatry. 1997;36(6):793-798.

21. Kaufman J, Yang BZ, Douglas-Palumberi H, et al. Social supports and serotonin transporter gene moderate depression in maltreated children. Proc Natl Acad Sci US A. 2004;101(49):17316-17321.

22. Browne A, Finkelhor D. Impact of child sexual abuse: a review of the research. Psychol Bull. 1986;99(1):66-77.

23. Romans SE, Martin JL, Anderson JC, Herbison GP, Mullen PE. Sexual abuse in childhood and deliberate self-harm. Am J Psychiatry. 1995;152(9):1336-1342.

24. Joiner TE, Jr., Sachs-Ericsson NJ, Wingate LR, Brown JS, Anestis MD, Selby EA. Childhood physical and sexual abuse and lifetime number of suicide attempts: a persistent and theoretically important relationship. Behav Res Ther. 2007;45(3):539-547.

25. Fergusson DM, Horwood LJ, Lynskey MT. Childhood sexual abuse and psychiatric disorder in young adulthood: II. Psychiatric outcomes of childhood sexual abuse. $J \mathrm{Am}$ Acad Child Adolesc Psychiatry. 1996;35(10):1365-1374.

26. Molnar BE, Berkman LF, Buka SL. Psychopathology, childhood sexual abuse and other childhood adversities: relative links to subsequent suicidal behaviour in the US. Psychol Med. 2001;31(6):965-977. 
27. Brent DA, Oquendo M, Birmaher B, et al. Familial pathways to early-onset suicide attempt: risk for suicidal behavior in offspring of mood-disordered suicide attempters. Arch Gen Psychiatry. 2002;59(9):801-807.

28. Melhem NM, Brent DA, Ziegler M, et al. Familial pathways to early-onset suicidal behavior: familial and individual antecedents of suicidal behavior. Am J Psychiatry. 2007;164(9):1364-1370.

29. Bradley SJ. Affect Regulation and the Development of Psychopathology. New York: The Guilford Press; 2003.

30. Kagan J. What is Emotion? History, Measures, and Meanings. New Haven, CT: Yale University Press; 2007.

31. Cole PM, Martin SE, Davis TA. Emotion regulation as a scientific construct: methodological challenges and directions for child development research. Child Development. 2004;75(2):317-333.

32. Mayer JD, Roberts RD, Barsade SG. Human abilities: emotional intelligence. Annu Rev Psychol. 2008;59:507-536.

33. Mayer JD, Salovey P, Caruso DR. Emotional intelligence: new ability or eclectic traits? Am Psychol. Sep 2008;63(6):503-517.

34. Salovey P, Mayer JD. Emotional intelligence. Imagination, Cognition, and Personality. 1990;9(3):185-211.

35. Salovey P, Sluyter DJ. Emotional Development and Emotional Intelligence: Educational Implications. New York, NY: Basic Books; 1997.

36. Ciarrochi J, Deane FP, Anderson S. Emotional intelligence moderates the relationship between stress and mental health. Pers Individ Dif. 2002;32:197-209. 
37. Nock MK, Banaji MR. Assessment of self-injurious thoughts using a behavioral test. Am J Psychiatry. 2007;164(5):820-823.

38. Nock MK, Mendes WB. Physiological arousal, distress tolerance, and social problemsolving deficits among adolescent self-injurers. J Consult Clin Psychol. 2008;76(1):2838.

39. Wedig MM, Nock MK. Parental expressed emotion and adolescent self-injury. J Am Acad Child Adolesc Psychiatry. 2007;46(9):1171-1178.

40. Bernstein DP, Ahluvalia T, Pogge D, Handelsman L. Validity of the Childhood Trauma Questionnaire in an adolescent psychiatric population. J Am Acad Child Adolesc Psychiatry. 1997;36(3):340-348.

41. Mayer JD, Salovey P, Caruso DR. MSCEIT YV: Mayer-Salovey-Caruso Emotional Intelligence Test: Youth Version, Research Version 1.0. Toronto, Canada: Multi-Health Systems; 2005.

42. Brackett MA, Mayer JD. Convergent, discriminant, and incremental validity of competing measures of emotional intelligence. Pers Soc Psychol Bull. 2003;29(9):11471158.

43. Mayer JD, Salovey P, Caruso DR, Sitarenios G. Measuring emotional intelligence with the MSCEIT V2.0. Emotion. 2003;3(1):97-105.

44. Brackett MA, Salovey P. Measuring emotional intelligence with the Mayer-SaloveyCaruso Emotional Intelligence Test (MSCEIT). Psicothema. 2006;18(Suppl):34-41.

45. Nock MK, Holmberg EB, Photos VI, Michel BD. Self-Injurious Thoughts and Behaviors Interview: development, reliability, and validity in an adolescent sample. Psychol Assess. 2007;19(3):309-317. 
46. Tabachnick BG, Fidell LS. Using Multivariate Statistics. 4th ed. Needham Heights, MA: Allyn \& Bacon; 2001.

47. Aiken LS, West SG. Multiple Regression: Testing and Interpreting Interactions. Newbury Park, CA: Sage; 1991.

48. Holmbeck GN. Post-hoc probing of significant moderational and mediational effects in studies of pediatric populations. J Pediatr Psychol. Jan-Feb 2002;27(1):87-96.

49. Baron RM, Kenny DA. The moderator-mediator variable distinction in social psychological research: conceptual, strategic, and statistical considerations. J Pers Soc Psychol. 1986;51(6):1173-1182.

50. Brown GK, Ten Have $T$, Henriques GR, Xie SX, Hollander JE, Beck AT. Cognitive therapy for the prevention of suicide attempts: a randomized controlled trial. JAMA. 2005;294(5):563-570.

51. Linehan MM, Comtois KA, Murray AM, et al. Two-year randomized controlled trial and follow-up of dialectical behavior therapy vs therapy by experts for suicidal behaviors and borderline personality disorder. Arch Gen Psychiatry. Jul 2006;63(7):757-766.

52. Lopes PN, Salovey P, Côté S, Beers M. Emotion regulation abilities and the quality of social interaction. Emotion. 2005;5(1):113-118.

53. Eack SM, Hogarty GE, Greenwald DP, Hogarty SS, Keshavan MS. Cognitive enhancement therapy improves emotional intelligence in early course schizophrenia: preliminary effects. Schizophr Res. 2007;89(1-3):308-311.

54. Hogarty GE, Greenwald DP. Cognitive Enhancement Therapy: The Training Manual: University of Pittsburg Medical Center; 2006. 
55. Spirito A, Stanton C, Donaldson D, Boergers J. Treatment-as-usual for adolescent suicide attempters: implications for the choice of comparison groups in psychotherapy research. $J$ Clin Child Adolesc Psychol. 2002;31(1):41-47.

56. Ciarrochi J, Blackledge J, Bilich L, Bayliss V. Improving emotional intelligence: a guide to mindfulness-based emotional intelligence training. In: Ciarrochi J, Mayer JD, eds. Applying Emotional Intelligence: A Practitioner's Guide. New York: Psychology Press; 2007.

57. Linehan MM, Goodstein JL, Nielsen SL, Chiles JA. Reasons for staying alive when you are thinking of killing yourself: the reasons for living inventory. J Consult Clin Psychol. $1983 ; 51(2): 276-286$.

58. Osman A, Downs WR, Kopper BA, et al. The Reasons for Living Inventory for Adolescents (RFL-A): development and psychometric properties. J Clin Psychol. 1998;54(8):1063-1078.

59. Osman A, Gutierrez PM, Muehlenkamp JJ, Dix-Richardson F, Barrios FX, Kopper BA. Suicide Resilience Inventory-25: development and preliminary psychometric properties. Psychol Rep. 2004;94(3 Pt 2):1349-1360.

60. Mestre JM, Guil R, Lopes PN, Salovey P, Gil-Olarte P. Emotional intelligence and social and academic adaptation to school. Psicothema. 2006;18 Suppl:112-117.

61. Lopes PN, Brackett MA, Nezlek JB, Schutz A, Sellin I, Salovey P. Emotional intelligence and social interaction. Pers Soc Psychol Bull. 2004;30(8):1018-1034.

62. Amitay OA, Mongrain M. From emotional intelligence to intelligent choice of partner. $J$ Soc Psychol. 2007;147(4):325-343. 
63. MacCann C, Matthews G, Zeidner M, Roberts RD. Psychological Assessment of Emotional Intelligence: A review of Self-Report and Performance-Based Testing. The International Journal of Organizational Analysis. 2003;11(3):247-274.

64. Widom CS, Morris S. Accuracy of adult recollections of childhood victimization: part 2. childhood sexual abuse. Psychol Assess. 1997;9:34-46.

65. Fergusson DM, Horwood LJ, Woodward LJ. The stability of child abuse reports: a longitudinal study of the reporting behaviour of young adults. Psychol Med. 2000;30(3):529-544.

66. Conte JR, Schuerman JR. Factors associated with an increased impact of child sexual abuse. Child Abuse Negl. 1987;11:201-211.

67. Finkelhor D, Browne A. The traumatic impact of child sexual abuse: a conceptualization. Am J Orthopsychiatry. 1985;55(4):530-541.

68. Briere J, Runtz M. Childhood sexual abuse: long-term sequelae and implications for psychological assessment. J Interpers Violence. 1993;8(3):312-330.

69. Martin G, Bergen HA, Richardson AS, Roger L, Allison S. Sexual abuse and suicidality: gender differences in a large community sample of adolescents. Child Abuse Negl. 2004;28:491-503. 
Table 1. Correlations among Suicidal Behaviors, CSA and EI Subscales

\begin{tabular}{|c|c|c|c|c|c|c|}
\hline Variable & 1 & 2 & 3 & 4 & 5 & 6 \\
\hline \multicolumn{7}{|c|}{ Suicidal Behaviors (Yr Freq) } \\
\hline 1. Suicide Ideation & - & & & & & \\
\hline 2. Suicide Attempt & $.62 * *$ & - & & & & \\
\hline \multicolumn{7}{|c|}{ Environmental Stressor } \\
\hline 3. CSA & $.35 * *$ & $.35 * *$ & - & & & \\
\hline \multicolumn{7}{|l|}{ Protective Factors } \\
\hline 4. EI Total & -.19 & -.12 & -.13 & - & & \\
\hline 5. Strategic EI & -.19 & -.19 & -.15 & $.89 * *$ & - & \\
\hline 6. Experiential EI & -.13 & -.02 & -.08 & $.86^{* *}$ & $.52 * *$ & - \\
\hline Mean $(S D)$ & $26.07(54.45)$ & $0.46(1.19)$ & $6.26(3.08)$ & $1.27(0.15)$ & $1.25(0.62)$ & $1.30(0.16)$ \\
\hline Range & $0-202$ & $0-5$ & $5-19$ & $0.76-1.47$ & $0.40-1.39$ & $1.06-1.64$ \\
\hline
\end{tabular}

Note $. \mathrm{CSA}=$ Childhood sexual abuse $; \mathrm{EI}=$ Emotional intelligence. Means and standard deviations for EI scores represent

item (i.e., not subscale) averages. ${ }^{*} p<.05 ; * *<.01$ 
Table 2. EI Moderates the Relation between CSA and Suicidal Behaviors

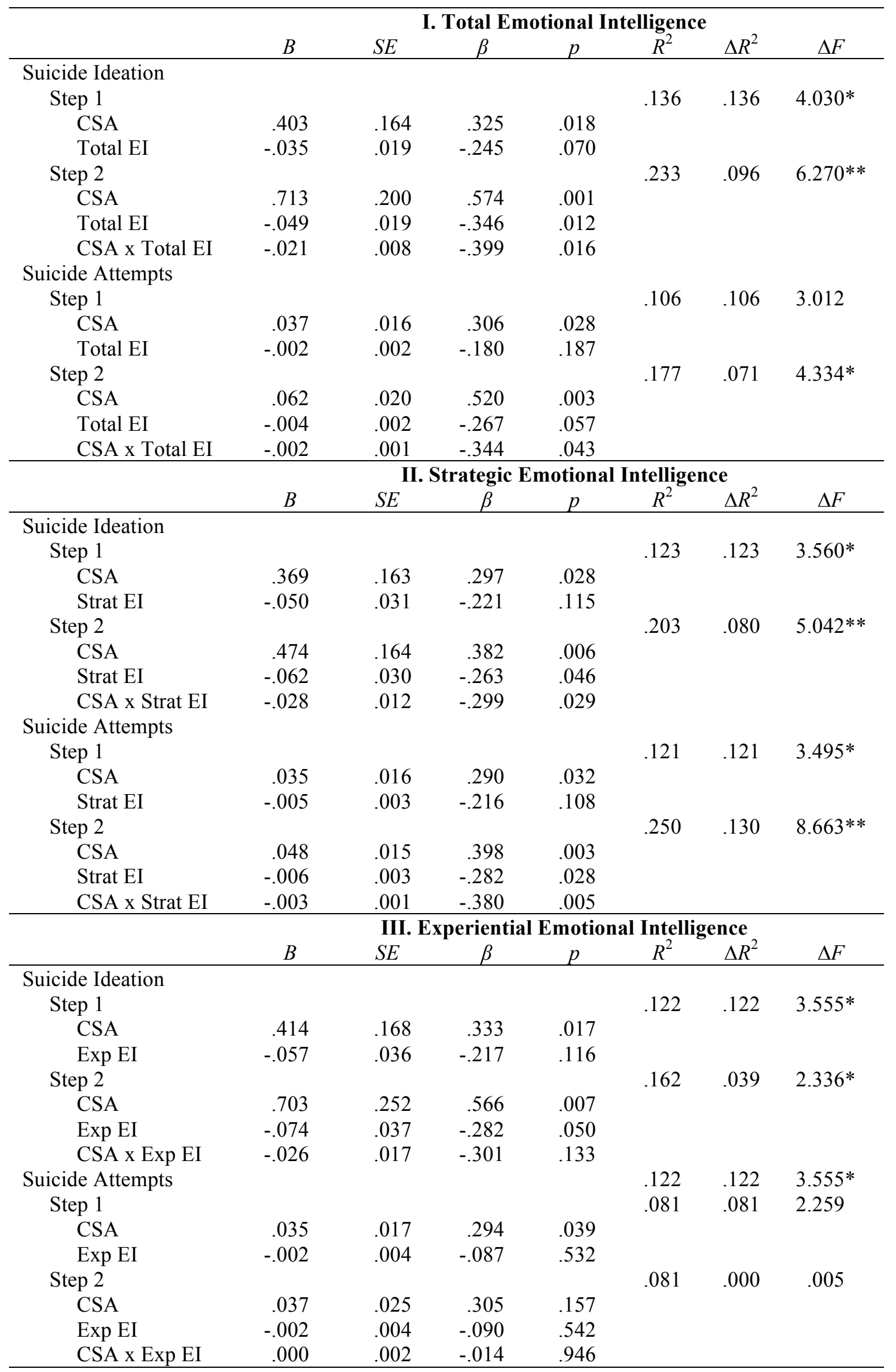


Note. $\mathrm{CSA}=$ Childhood sexual abuse $; \mathrm{EI}=$ Emotional intelligence; Strat $\mathrm{EI}=$ Strategic emotional intelligence; Exp EI $=$ Experiential emotional intelligence. ${ }^{*} p<.05,{ }^{* *} p<.01$ 


\section{Figure Captions}

Figure 1a. Total EI Moderates the Relations between CSA and Suicidal Behaviors

Figure 1b. Strategic EI Moderates the Relations between CSA and Suicidal Behaviors

Figure 1c. Experiential EI Does Not Moderate the Relations between CSA and Suicidal

Behaviors

Note. $\mathrm{SI}=$ Suicide ideation $; \mathrm{SA}=$ Suicide attempts; $\mathrm{EI}=$ Emotional intelligence; $\mathrm{StratEI}=$

Strategic emotional intelligence; ExpEI $=$ Experiential emotional intelligence. Coefficients from simple slope analyses correspond to each plotted line. ${ }^{*} p<.05,{ }^{* *} p<.01,{ }^{* * *} p<.001$ 
Fig. 1a

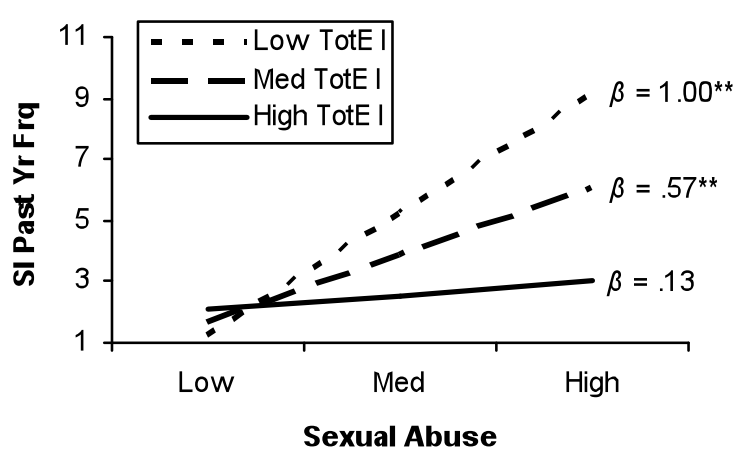

Fig. 1b

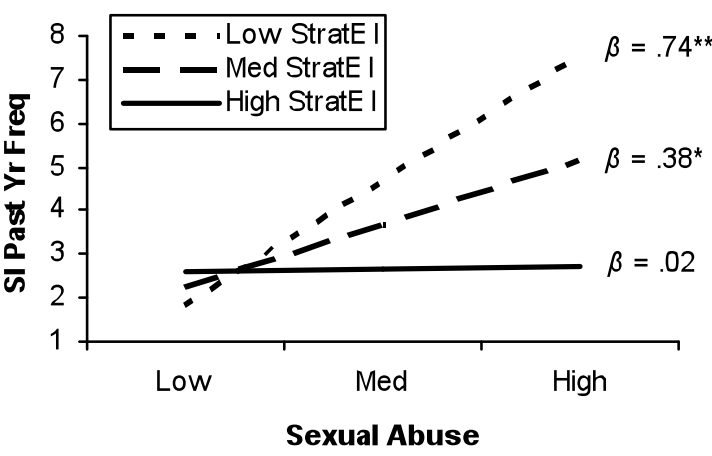

Fig. 1c
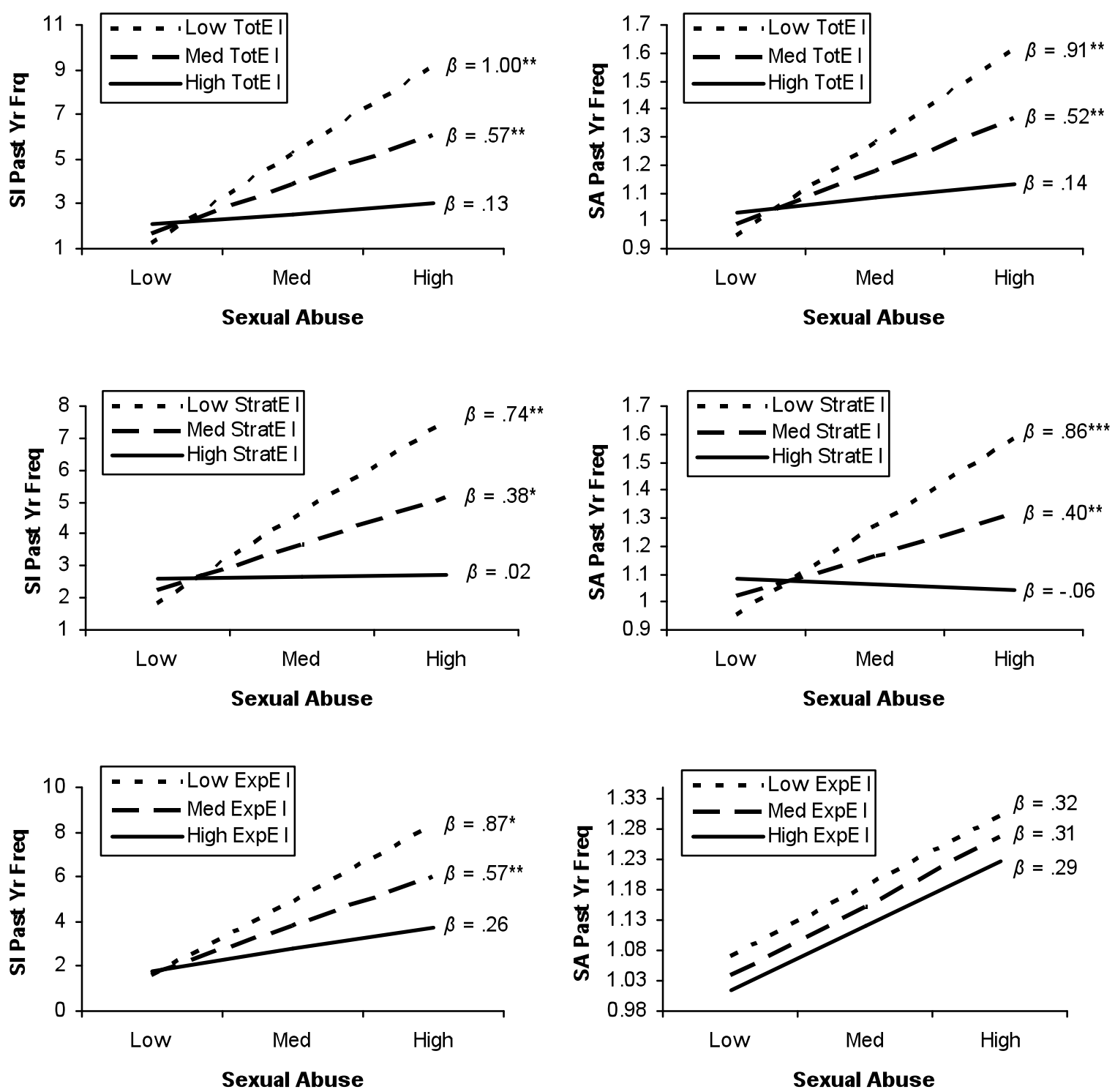